\title{
A scalable solid-state quantum computer based on quantum dot pillar structures
}

\author{
G. D. Sanders, K. W. Kim, and W. C. Holton \\ Department of Electrical and Computer Engineering, North Carolina State University \\ Raleigh, North Carolina 27695-7911
}

\begin{abstract}
We investigate an optically driven quantum computer based on electric dipole transitions within coupled single-electron quantum dots. Our quantum register consists of a freestanding n-type pillar containing a series of pair wise coupled asymmetric quantum dots, each with a slightly different energy structure, and with grounding leads at the top and bottom of the pillar. Asymmetric quantum wells confine electrons along the pillar axis and a negatively biased gate wrapped around the center of the pillar allows for electrostatic confinement in the radial direction. We self-consistently solve coupled Schrödinger and Poisson equations and develop a design for a threequbit quantum register. Our results indicate that a single gate electrode can be used to localize a single electron in each of the quantum dots. Adjacent dots are strongly coupled by electric dipole-dipole interactions arising from the dot asymmetry, thus enabling rapid computation rates. The dots are tailored to minimize dephasing due to spontaneous emission and phonon scattering and to maximize the number of computation cycles. The design is scalable to a large number of qubits.

PACS Number(s): 03.67.Lx, 73.20.Dx, 85.30.Vw
\end{abstract}

Typeset using REVTEX 


\section{INTRODUCTION}

The possibility that a computer with exceptional properties could be built employing the laws of quantum physics has stimulated considerable interest in searching for useful algorithms and realizable physical implementations. Two useful algorithms, exhaustive search 1 and factorization, 2 have been discovered; others, including the suggestion that quantum computers will prove useful to model quantum systems, are being sought. Meanwhile, various physical implementations are being explored, including trapped ions, 3 cavity quantum

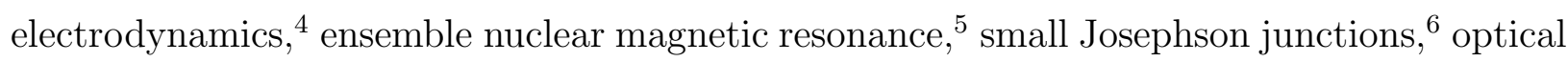
devices incorporating beam splitters and phase shifters based on quantum dots. for particular key applications, the requirements for their implementation are extremely stringent, perhaps especially rigorous for solid-state systems. Nevertheless solid-state quantum computers are very appealing relative to other possible implementation schemes because of the well-known ability to customize the design through the use of artificially structured materials and the probable scalability of the resulting design. For example, integrated circuit manufacturing technology would be immediately applicable to quantum computers of the proper implementation; and such designs would not only be scalable to smaller dimensions along the "semiconductor learning curve" but also large ensembles of "identical" quantum computers could be manufactured, that could be individually fine-tuned electrically. To date, no solid-state implementation of quantum computing has been demonstrated.

In this paper, we investigate a solid-state quantum computer implementation that is amenable to manufacturing with integrated circuit technology. We develop a threedimensional (3D) device model and self-consistently solve coupled Schrödinger and Poisson equations to generate a quantum computer design for a three-qubit quantum register that is based on pair wise coupled asymmetric III-V quantum dots. The design is optimized for a long coherence time and a rapid computation rate. Our results indicate that this structure may provide a realistic scalable candidate for quantum computing in solid-state systems. 


\section{PROPOSED STRUCTURE}

The proposed quantum dot quantum computer (see Fig. 1) consists of a pillar structure composed of a chain of asymmetric quantum dots separated by intervening layers of higher bandgap composition fabricated in a GaAs/AlGaAs technology by means of a sequence of planar MBE growth steps and subsequent etching to form the pillar. A sheath of similar AlGaAs composition is then grown surrounding the pillar and a wrap-around gate electrode deposited. A drain (source) is formed at the top (bottom), the series of asymmetric quantum dots are in the center region, and the gate surrounds the region of the pillar containing the quantum dots. Tarucha et al. 13 have reported similar n-type single electron transistor (SET) structures. Electron confinement along the pillar axis is produced by the band gap discontinuity of the dot structure. Encasing the quantum dot structure in the pillar core by the cylindrical sheath and the gate electrode provides confinement in the radial direction. By applying a negative bias that depletes carriers near the surface, an additional parabolic electrostatic potential is formed that allows for tuning of the radial confinement and localization of one electron per dot. The simultaneous insertion of a single electron per dot is accomplished by lining up the quantum dot ground state levels so that they lie close to the Fermi level; a single electron is confined in each dot over a finite range of the gate voltage due to shell filling effects.13

Thus, the pillar consists of a vertical stack of coupled asymmetric GaAs/AlGaAs quantum dots of differing size and composition so that each dot possesses a distinct energy structure. Qubit registers, $|0\rangle$ and $|1\rangle$, are based on the ground and first excited state of the single electron within each quantum dot. Overall, parameters of the structure can be chosen to produce a well-resolved spectrum of distinguishable qubits. The asymmetric dots produce large built-in electrostatic dipole moments between the ground and first excited state, and electrons in adjacent dots are coupled through the electric dipole-dipole interaction, while coupling between non-adjacent dots is significantly weaker. This produces the desired quantum computer consisting of a linear array of binary states (qubits) with 
pair wise pillar-axis coupling between adjacent qubits.14 In addition to energy tuning, the asymmetry of each quantum dot can be designed so that dephasing due to electron-phonon scattering and spontaneous emission is minimized. The combination of strong dipole-dipole coupling and long dephasing times make it possible to perform many computational steps before loss of coherence, in fact, it is believed possible to design this device so that error correction substantially prohibits coherence loss.

Quantum computations are performed by means of a series of coherent optical pulses in the far infrared. Final readout of the amplitude and phase of the qubit states can be achieved through quantum state holography. Amplitude and phase information are extracted through mixing the final state with a reference state generated in the same system by an additional delayed laser pulse and detecting the total time- and frequency-integrated fluorescence as a function of the delay. 15 Extracting the final state information using quantum state holography requires multiple experiments, one for each delay, as described in Ref. 15. Thus, the computation must be performed several times before an answer is arrived at. This is no real problem since the number of repetitions needed is only on the order of 40 or so, independent of the number of computational steps in a given quantum algorithm. Through the use of integrated circuit manufacturing technology, it is possible to simultaneously fabricate a large array of "identical" pillar quantum dot quantum computers, that is, on the order of $10^{10}$ per wafer. Each of these quantum registers could be electrically connected through deposited interconnect in such a manner so that each could be individually tunable to produce an array of identical units. In general, inhomogeneity among the quantum dots will result in slightly different energy levels. Sherwin et al. 16 have recently pointed out that one can perform accurate qubit operations in an inhomogeneous population of quantum dots arising from quenched disorder due to static charged defects, for example, provided that each SET is independently calibrated. This calibration can done by performing simple gate operations and tuning the gate electrodes appropriately. Efficient optical coupling to the resulting ensemble can be achieved through optical light guiding as suggested in Ref. 17. By this means direct observation of fluorescence is possible. Quantum computations are per- 
formed by means of a series of coherent optical pulses in the far infrared, and may be carried

out in complete analogy with the operation of an NMR quantum computer 18 It should be remarked that while our scheme resembles NMR ensemble quantum computation in the use of a series of optical pulses to perform quantum logic gates, it differs from NMR quantum computation in that our use of a collection of single electron transistors is done to enable a stronger signal to noise ratio in the readout phase. In principle, the quantum computation could be done with only a single SET transistor structure if the readout measurements were sufficiently sensitive.

\section{DEVICE MODEL}

In the context of studies of the Coulomb blockade in self-organized quantum dots and planar single-electron transistors, self-consistent calculations of electronic structure, shell filling effects, electron-electron interaction, Coulomb degeneracy, and Coulomb oscillation amplitudes have been carried out for various quantum dot structures.19 27 Our quantum register can be analyzed using methods similar to those used to study the self-consistent electronic structure in single-electron transistors. The problem we address is similar to those addressed by other authors who are interested in obtaining current-voltage characteristics and studying Coulomb oscillations in single-electron transistors over a wide range of gate biasing and shell filling conditions. 1927

In our case, we are interested in obtaining the self-consistent electrostatic potential and electronic eigenstates in an equilibrium configuration in which the source and drain are grounded and the gate electrode is negatively biased. The electrostatic potential, $V(r)$, is obtained by solving the Poisson equation for n-doped semiconductors22.27

$$
\nabla^{2} V(\vec{r})=-\frac{4 \pi}{\varepsilon} q\left[n(\vec{r})+N_{D}^{+}(\vec{r})\right]
$$

In the Poisson equation, $q$ is the absolute value of the electron charge, $\varepsilon$ is the static dielectric constant, $n(\vec{r})$ is the electron concentration and $N_{D}^{+}(\vec{r})$ is the known concentration of ionized 
donors in the structure. For the dielectric constant, we adopt the GaAs value $\varepsilon=12.28$ The Poisson equation is solved subject to boundary conditions on the electrostatic potential, $V(\vec{r})$. At the interfaces between the semiconductor and the source, drain and gate electrodes, $V(\vec{r})$ is equal to the applied gate voltage while at the semiconductor-vacuum interfaces, the normal derivative of $V(\vec{r})$ vanishes.

Following Ref. 22, the global electron concentration, $n(\vec{r})$, in the device is obtained by partitioning the pillar structure into "bulk" and "quantum" regions. In the "bulk" regions far from the quantum wells i.e. the source and drain regions, electrons are treated in the Thomas-Fermi approximation and the electron concentration is given by 29

$$
n(\vec{r})= \begin{cases}\frac{1}{3 \pi^{2}}\left[\frac{2 m_{e}^{*}}{\hbar^{2}}(\mu-U(\vec{r}))\right]^{3 / 2} & \text { if } U(\vec{r})<\mu \\ 0 & \text { otherwise }\end{cases}
$$

where $\mu$ is the chemical potential and $U(\vec{r})$ is the effective electron potential. The chemical potential, $\mu$, is determined through the requirement that overall charge neutrality be maintained in the bulk regions, i.e. the chemical potential is adjusted until

$$
\int\left(n(\vec{r})-N_{D}^{+}(\vec{r})\right) d \vec{r}=0
$$

where the integration is carried out over the bulk source and drain regions.

The effective potential, $U(\vec{r})$, in the bulk regions includes the Hartree potential, $U_{H}=$ $-q V(\vec{r})$, and the conduction band offset, $\Delta E_{c}$, which depends on the local Al concentration, $x$. Thus,

$$
U(\vec{r})=-q V(\vec{r})+\Delta E_{c}
$$

where the conduction band offset, $\Delta E_{c}$, is taken to be $60 \%$ of the difference between the $A l_{x} G a_{1-x} A s$ and $G a A s$ bandgaps. Using the bandgap variation of $A l_{x} G a_{1-x} A s$ determined by Lee et al. 30 we obtain the following expression for the conduction band offset as a function of the local Al concentration, $x$ :

$$
\Delta E_{c}=0.6\left(1155 x+370 x^{2}\right) \mathrm{meV}
$$


In the "quantum" regions containing the quantum wells, the electron concentration, $n(r)$, is determined by the electron wavefunctions, $\psi_{i}(r)$, and energies, $E_{i}$, through the relation

$$
n(\vec{r})=\sum_{i} n_{i}\left|\psi_{i}(\vec{r})\right|^{2}
$$

The electron occupancy in each level, $n_{i}$, is a function of the electron energy and the temperature.

The electron wavefunctions and energy levels, $E_{i}$, are obtained by solving the Schrödinger equation in the effective mass approximation

$$
\left[-\frac{\hbar^{2}}{2 m_{e}^{*}} \nabla^{2}+U(\vec{r})-E_{i}\right] \psi_{i}(\vec{r})=0
$$

The electron potential, $U(\vec{r})$, in the quantum regions is given by

$$
U(\vec{r})=-q V(\vec{r})+\Delta E_{c}+U_{x c}(\vec{r})
$$

where $U_{x c}(\vec{r})$ is the exchange-correlation potential of Perdew and Zunger. 11

In the quantum register discussed in the next section, the gate voltage is negatively biased in such a way that a single electron is strongly localized in each electrostatically confined quantum dot. The radial confinement potential is strong enough that the lowest few electron wavefunctions are strongly localized near the center of the pillar and die away far from the semiconductor-electrode interface. In our design, the quantum wells are wide enough and the barriers between the quantum wells are thick enough so that the lowest few electron wavefunctions do not penetrate to the center of the barriers separating the quantum wells. Since all the wavefunctions of interest vanish at the center of these barriers, we can divide the quantum well region into several regions (one for each qubit). These regions are taken to be cylinders stacked along the pillar axis with top and bottom surfaces located at the centers of the barriers between adjacent wells. We solve the Schrödinger equation in each dot separtely subject to the boundary condition that all wavefunctions vanish at the region boundaries.

Due to the cylindrical symmetry of the structure, the 3D Schrödinger equation can be reduced to a $2 \mathrm{D}$ equation in cylindrical coordinates. One might try to solve the $2 \mathrm{D}$ 
Schrödinger equation by finite differencing the partial differential equation and solving the resulting matrix eigenvalue equation. The size of the matrix to be diagonalized is equal to the number of interior mesh points in the $2 \mathrm{D}$ grid and this is much too large to be handled easily. Other authors have taken this brute-force approach to solving the Schrödinger equation in self-consistent Poisson-Schrödinger problems with the result that solving the Schrödinger equation is the most time consuming part of the computation.22 We find that it is possible to do better. In solving the $2 \mathrm{D}$ Schrödinger equation, we first approximate $U(\rho, z)$ in each quantum dot by a separable potential

$$
U(\rho, z) \approx U_{s}(\rho, z) \equiv U_{r}(\rho)+U_{z}(z)
$$

where the axial potential is defined as

$$
U_{z}(z)=\frac{2}{R^{2}} \int_{0}^{R} U(\rho, z) \rho d \rho
$$

and the radial potential is given by

$$
U_{r}(\rho)=\frac{1}{\mathcal{L}} \int_{0}^{\mathcal{L}}\left(U(\rho, z)-U_{z}(z)\right) d z
$$

In these last two expressions, $R$ and $\mathcal{L}$ are the radius and height of the cylindrical region over which $U(\rho, z)$ is defined in each dot. With the separable potential approximation, the 2D Schrödinger equation can be separated into two $1 \mathrm{D}$ equations which can be cast as finite difference eigenvalue equations and solved numerically for the electron energies and wavefunctions. The resulting $2 \mathrm{D}$ wavefunctions are the best product wavefunctions that approximate the solution of the 2D Schrödinger equation in each qubit. The electronic states in the separable potential approximation in our cylindrical pillar are labeled by three quantum numbers $\left(n_{\rho}, n_{\phi}, n_{z}\right)$ which specify the number of nodes in the product wavefunctions associated with cylindrical coordinates $\rho, \phi$, and $z$. In this notation, the qubit state $|0\rangle$ is denoted $(0,0,0)$ while $|1\rangle$ is denoted $(0,0,1)$. We find that the separable wavefunctions are reasonable approximations to the true wavefunctions since we are starting with a separable potential which is already close to the true potential in some average sense. We next obtain 
the exact energies and wavefunctions of the original non-separable Schrödinger equation by treating the residual $U(\rho, z)-U_{s}(\rho, z)$ as a perturbation and expanding the exact wavefunctions as a sum of separable wavefunctions. Our expansion of the true wavefunctions in terms of separable wavefunctions is rapidly converging and we find that the dominant terms in the expansion of the true wavefunctions are the separable wavefunctions of the same symmetry. Our approach to solving the 2D Schrödinger equation is fast and most of computing time is spent solving the Poisson equation.

To complete the specification of the electron charge density in the quantum dots, it is necessary to compute the electron occupation numbers, $n_{i}$. One might expect that $n_{i}$ would be given by the Fermi-Dirac distribution and indeed this would be the case if the electrons in the dots were delocalized and in tunneling contact with the leads. In this case, the qubits could exchange electrons with their environment and the total number of electrons in the dot $N=\sum_{i} n_{i}$ could take on non-integer values. But clearly this is not tolerable in a quantum computer and we must carefully arrange things so the dot wavefunctions exhibit a high degree of localization. In this situation, only an integer number of electrons can occupy the dot and this constraint gives rise to what is known as the Gibbs distribution. The number of electrons, $N$, in the dot is determined by minimizing the Gibbs free energy with respect to the integer number of electrons, $N$. The Gibbs free energy is $F(N)=-k T \ln [Z(N)]$, where the grand canonical partition function, $Z(N)$, is given by 22,26

$$
Z(N)=\sum_{\left\{n_{i}\right\}} \exp \left[\frac{\sum_{i} n_{i} E_{i}-E_{H}(N)-\mu N}{k T}\right]
$$

The lack of diffusive contact between the quantum dots and the rest of the device means that the chemical potential, $\mu$, is determined by electrons in the leads and contacts. The summation in $Z(N)$ is carried out over all electron configurations $\left\{n_{i}\right\}$ for which $\sum_{i} n_{i}=N$. Double counting the Coulombic interaction is avoided by subtracting the Hartree energy $E_{H}(N)$ for the $N$ electrons. The Hartree energy appearing in the partition function is 26

$$
E_{H}(N)=\frac{1}{8 \pi \varepsilon} \int \frac{n_{e}(\vec{r}) n_{e}\left(\vec{r}^{\prime}\right)}{\left|\vec{r}-\vec{r}^{\prime}\right|} d \vec{r} d \vec{r}^{\prime}
$$


where $n_{e}(\vec{r})$ is the charge in the quantum dot and the integration is restricted to the dot region. Directly solving for the Hartree energy by performing a double integral over the quantum dot charge density is too time consuming and impractical due to the presence of the singularity in the integrand at $\vec{r}=\vec{r}^{\prime}$. An alternative method of calculating the Hartree energy is to use the equivalent expression

$$
E_{H}(N)=\frac{1}{8 \pi \varepsilon} \int V_{e}(\vec{r}) n_{e}(\vec{r}) d \vec{r}
$$

where the potential $V_{e}(\vec{r})$ is obtained by solving the Poisson equation in the pillar using the charge density, $n_{e}(\vec{r})$, in the quantum dot.27

The boundary condition on $V_{e}(\vec{r})$ at the surface of the pillar is determined by asymptotically expanding $V_{e}(\vec{r})$ in a multipole expansion in the quantum dot charge density up through quadrupole terms and using this expansion to specify $V_{e}$ at the surface. This is a good approximation since the pillar boundaries are far from the localized quantum dot charge.2.

To obtain a self-consistent solution to the coupled Poisson and Schrödinger equations, we first specify the device structure including the $A l_{x} G a_{1-x} A s$ alloy composition, the doping concentration, and the arrangement of the electrodes. In all our runs, the source and drain are assumed to be grounded and the gate is assumed to be negatively biased. We initially assume complete depletion in the structure and solve the Poisson equation to obtain an initial guess for the electrostatic potential, $V(\rho, z)$. With this electrostatic potential and the quantum well band offset potentials, we solve the Schrödinger equation for the unoccupied quantum dot energies and wavefunctions. The chemical potential in the depleted structure is set to the minimum of the Thomas-Fermi electron potential, $U=-q V(\rho, z)+\Delta E_{c}$, in the source and drain regions. Starting with these initial guesses for the chemical potential in the leads and the solutions of the Poisson and Schrödinger equations, we obtain self-consistent solutions through the following relaxation procedure.

First, electron densities in the leads and the quantum dots are obtained from the chemical potential, the temperature, and the quantum dot electronic states. The global charge density, 
including the given doping charge, is then obtained. Next the Poisson equation is solved for $V(\rho, z)$. The Hartree potential and exchange-correlation potentials are then obtained from $V(\rho, z)$ and the electron charge density. With the electron potentials in hand, the Schröodinger equation is solved in each quantum dot region. The procedure is then repeated until convergence is achieved. In updating the electrostatic potential and electron charge density, the new solutions are mixed with the old to obtain the updated solutions. For the electrostatic potential

$$
V(\rho, z) \rightarrow \lambda V_{\text {new }}(\rho, z)+(1-\lambda) V_{\text {old }}(\rho, z)
$$

where $\lambda<1$ is a relaxation parameter which is dynamically adjusted to accelerate conver-

gence. A similar scheme is used to update the electron charge density. The above procedure is iterated until the chemical potential, electrostatic potential, electron charge density, and quantum dot energy levels all change by less than some small relative tolerance between successive iterations at which point convergence is achieved. Typically about 400 iterations are required to achieve convergence to within one part in $10^{4}$.

\section{A THREE QUBIT QUANTUM REGISTER: 1D ANALYSIS}

We can use the device modeling program described in the last section to obtain a design for a three-qubit quantum register. We could, in principle, do a full 3D analysis of the device and obtain suitable design parameters (i.e., pillar dimensions, doping concentrations, asymmetric well shapes, electrode placement and biasing, etc.) based on our computationally intensive 3D model. Clearly this would be prohibitively time consuming due to the size of the parameter space that would need to be investigated as well as the time required to perform each run. To narrow down the design parameters, we can take advantage of the fact that our quantum computer is operated in the extreme depletion limit and do a simple 1D analysis to gain some useful insight.

Let's assume that inside the core of stacked quantum wells (radius $R_{c}$ ) we have complete depletion and uniform doping. In this limit, the quantum dot electron potential, $U(r)$, can be 
expressed in cylindrical coordinates as $U(\vec{r})=U(z)+U(\rho)$, where $U(\rho)$ is a radial potential arising from the uniform donor density and $U(z)$ is the conduction band offset potential along the growth direction. This separable potential assumption is a good approximation in the strong depletion regime where only a single electron resides in each dot. The assumption of a separable potential is commonly used in the study of quantum dot structures and enables us to consider the $z$ and $\rho$ motions separately. 13,32 The z-directional potential $U(z)$, shown schematically in the inset of Fig. 2, is a step potential formed by a layer of $A l_{x} G a_{1-x} A s$ of thickness $B(0<z<B)$ and a layer of GaAs of thickness $L-B(B<z<L)$. The resulting asymmetric quantum dot/well is confined by $A l_{y} G a_{1-y} A s$ barriers with $y>x$ and the asymmetry is parameterized by the ratio $B / L$ where $0<B / L<1$.

In the effective mass approximation, the qubit wavefunctions are $|i\rangle=R(\rho) \psi_{i}(z) u_{s}(\vec{r})$ ( $i=0,1)$. Here $R(\rho)$ is the ground state of the radial envelope function, $\psi_{i}(z)$ is the envelope function along $z$, and $u_{s}(\vec{r})$ is the $s$-like zone center Bloch function including electron spin. For simplicity, we assume complete confinement by the $A l_{y} G a_{1-y} A s$ barriers along the $\mathrm{z}$ direction. Then, the envelope function $\psi_{i}(z)$ is obtained by solving the time-independent Schrödinger equation subject to the boundary conditions $\psi_{i}(0)=\psi_{i}(L)=0$. The energies of the qubit wavefunctions are given by $E=E_{\rho}+E_{i}$ where $E_{\rho}$ is the energy associated with $R(\rho)$ and $E_{i}$ is the energy associated with $\psi_{i}(z)$.

Figure 2 shows the probability density, $\left|\psi_{i}(z)\right|^{2}$, as a function of position, $z$, for the two qubit states $|0\rangle$ and $|1\rangle$ in a $20 \mathrm{~nm} \mathrm{GaAs} / \mathrm{Al}_{0.3} \mathrm{Ga}_{0.7} \mathrm{As}$ asymmetric quantum dot. The barrier thickness $B=15 \mathrm{~nm}$ and the overall length of the dot is $L=20 \mathrm{~nm}$. By choosing $B / L=$ 0.75 and $x=0.3$, it is found that the ground state wavefunction $|0\rangle$ is strongly localized in the $G a A s$ region while the $|1\rangle$ wavefunction is strongly localized in the $A l_{0.3} G a_{0.7} A s$ barrier. By appropriately choosing the asymmetric quantum dot parameters, the qubit wavefunctions can be spatially separated and a large difference in the electrostatic dipole moments can be achieved.

The transition energy $\Delta E_{0}=E_{1}-E_{0}$ between $|1\rangle$ and $|0\rangle$ is shown in Fig. 3 as a function of $B / L$ in a $20 \mathrm{~nm} \mathrm{GaAs} / \mathrm{Al}_{x} G a_{1-x} A s$ asymmetric quantum $\operatorname{dot}(L=20 \mathrm{~nm})$. 
Several values of $\mathrm{Al}$ concentration $x$ are considered. In Fig. 4 , we fix the $\mathrm{Al}$ concentration at $x=0.2$ and plot $\Delta E_{0}$ as a function of $L$ for several values of $B / L$. The continuous curves are based on our 1D analysis and the squares are the qubit energy gaps for a three qubit self-consistent quantum register calculation as described in the next section. It is clear that the transition energy can be tailored substantially by varying the asymmetry parameter. With three parameters available for adjustment $(B, L$, and $x)$, we can make $\Delta E_{0}$ unique for each dot in the register. In this way, we can address a given dot by using laser light with the correct photon energy.

It is desirable that the $|1\rangle$ state be the first excited level of the quantum dot. Thus, the lowest lying radial state $(0,1,0)$ should lie above the $|1\rangle$ state. The radial energy gap, $\Delta E_{1}$, between the ground state, $|0\rangle$, and the first radial excited state, $(0,1,0)$, is found by solving a 2D Schrödinger equation for an electron in the radial potential, $U_{r}(\rho)$. If we take the barrier in the sheath to be infinite, then in the extreme depletion limit, we have

$$
U_{r}(\rho)= \begin{cases}-q V(\rho) & \text { if } \rho<R_{c} \\ \infty & \text { otherwise }\end{cases}
$$

where $V(\rho)$ is the radial electrostatic potential. For complete depletion and uniform doping, the Poisson equation for $V(\rho)$ can be solved analytically. Thus,

$$
V(\rho)=\frac{\pi N_{D}^{+}}{\varepsilon}\left(R_{c}^{2}-\rho^{2}\right)
$$

where $R_{c}$ is the sheath radius and $N_{D}^{+}$is the doping density. Numerically solving the 2D Schrödinger equation for an electron in the potential, $U_{r}(\rho)$, is straightforward. Figure 5 shows the radial energy gap, $\Delta E_{1}$, between the $|0\rangle$ and the lowest lying radial state, $(0,1,0)$, as a function of doping concentration, $N_{D}^{+}$, for several values of $R_{c}$. For narrow pillars with low doping concentrations, the radial energy gap is determined by size confinement. For large pillars with high doping concentrations the radial energy gap is determined by electrostatic confinement. From Fig. \&, we see that the qubit energy gaps reach a minimum near $\Delta E_{0} \sim 70 \mathrm{meV}$ for quantum wells with $L \sim 20 \mathrm{~nm}$. The results of Fig. 5 suggest that 
radial gaps in the range of $\Delta E_{1} \sim 100 \mathrm{meV}$ with strong size confinement can be achieved with doping densities in the range of $N_{D}^{+} \sim 10^{17} \mathrm{~cm}^{-3}$ if $R_{c} \sim 70 \stackrel{\circ}{A}$.

The electric field from an electron in one dot shifts the energy levels of electrons in adjacent dots through electrostatic dipole-dipole coupling. By appropriate choice of coordinate systems, the dipole moments associated with $|0\rangle$ and $|1\rangle$ equal in magnitude but oppositely directed. The dipole-dipole coupling energy is then defined as 8

$$
V_{d d}=2 \frac{\left|d_{1}\right|\left|d_{2}\right|}{\epsilon_{r} R_{12}^{3}}
$$

where $d_{1}$ and $d_{2}$ are the ground state dipole moments in the two dots, $\epsilon_{r}=12.9$ is the dielectric constant for $G a A s$, and $R_{12}$ is the distance between the dots.

Figure 6 shows the dipole-dipole coupling energy, $V_{d d}$, between two asymmetric GaAs $/ \mathrm{Al}_{x} G a_{1-x} A s$ quantum dots of widths $L 1=19 \mathrm{~nm}$ and $L 2=21 \mathrm{~nm}$ separated by a $10 \mathrm{~nm} A l_{y} G a_{1-y} A s$ barrier. The coupling energy is plotted as a function of $B / L$ for several values of $x$ where $B / L$ and $x$ are taken to be the same in both dots. The dipole-dipole coupling energies are a strongly peaked function of the asymmetry parameter, $B / L$. From the figure, we see that values of $V_{d d} \sim 0.15 \mathrm{meV}$ can be achieved.

Quantum dot electrons can interact with the environment through the phonon field, particularly the longitudinal-optical (LO) and acoustic (LA) phonons. The LO phonon energy, $\hbar \omega_{L O}$, lies in a narrow band around $36.2 \mathrm{meV}$. As long as the quantum dot energy level spacings lie outside this band, LO phonon scattering is strongly suppressed by the phonon bottleneck effect. Acoustic phonon energies are much smaller than the energy difference, $\Delta E$, between qubit states. Thus, acoustic phonon scattering requires multiple emission processes which are also very slow. Theoretical studies on phonon bottleneck effects in GaAs quantum dots indicate that LO and LA phonon scattering rates including multiple phonon processes could be slower than the spontaneous emission rate provided that the quantum dot energy level spacing is greater than $\sim 1 \mathrm{meV}$ and, at the same time, avoids a narrow window around the LO phonon energy.33 35 In Ref. 33, Inoshita and Sakaki compute multi-phonon relaxation rates in spherical single-electron GaAs quantum dots due to one- and two-phonon 
scattering by LO and LA phonons at $T=0 \mathrm{~K}$ and $T=300 \mathrm{~K}$. Using the results of this calculation, we estimate that multi-phonon scattering dominates the spontaneous emission only if the qubit energy level spacing is within $\sim 4 \mathrm{meV}$ of the LO phonon energy. Likewise, multi-phonon LA scattering becomes important if the qubit energy gaps are smaller than $\sim 1 \mathrm{meV}$

While dephasing via interactions with the phonon field can be strongly suppressed by proper designing of the structure, quantum dot electrons are still coupled to the environment through spontaneous emission and this is the dominant dephasing mechanism. Decoherence resulting from spontaneous emission ultimately limits the total time available for a quantum computation. 36 Thus, it is important that the spontaneous emission lifetime be large. The excited state lifetime, $T_{d}$, against spontaneous emission is 36

$$
T_{d}=\frac{3 \hbar(\hbar c)^{3}}{4 e^{2} D^{2} \Delta E^{3}}
$$

where $D=\langle 0|z| 1\rangle$ is the dipole matrix element between $|0\rangle$ and $|1\rangle$.

Figure 7 shows the spontaneous emission lifetime of an electron in qubit state $|1\rangle$ for a $20 \mathrm{~nm} \mathrm{GaAs} / \mathrm{Al}_{x} G a_{1-x} A s$ quantum dot as a function of asymmetry parameter, $B / L$, for several values of $\mathrm{Al}$ concentration, $x$. It is immediately obvious from Fig. 7 that the lifetime depends strongly on $B / L$. Depending on the value of $x$ chosen, the computed lifetime can achieve a maximum of between 4000 ns and 6000 ns. In general, the maximum lifetime increases with $x$. In Eq. (19), the lifetime is inversely proportional to $\Delta E^{3}$ and $D^{2}$, but the sharp peak seen in Fig. 7 is due primarily to a pronounced minimum in $D$.

Based on these results, we can estimate parameters for a solid state quantum register containing a stack of several asymmetric $G a A s / A l_{0.3} G a_{0.7} A s$ quantum dots in the $L \sim 20 \mathrm{~nm}$ range separated by $10 \mathrm{~nm} A l_{y} G a_{1-y} A s$ barriers $(y>0.4)$. An important design goal is obtaining a large spontaneous emission lifetime and a large dipole-dipole coupling energy. From Figs. 6 and 7, we see that both can be achieved by selecting an asymmetry parameter, $B / L=0.8$. This gives us a spontaneous emission lifetime $T_{d}=3100 \mathrm{~ns}$ and a dipole-dipole coupling energy $V_{d d}=0.14 \mathrm{meV}$. The transition energy between the qubit states is on 
the order of $100 \mathrm{meV}(\lambda=12.4 \mu \mathrm{m})$. In a quantum computation, the quantum register is optically driven by a laser as described in Ref. 8. In our example, we require a tunable infra-red laser in the mid-10 $\mu \mathrm{m}$ range so we can individually address various transitions between coupled qubit states.

\section{A THREE QUBIT QUANTUM REGISTER: 3D ANALYSIS}

Using the results of our simple 1D model as a starting point, we designed a three qubit quantum register by using the self-consistent device model described in Section III. Several criteria have to be met for a viable quantum register design and the structure we obtained through trial-and-error involved tradeoffs between several design goals.

For a self-consistent quantum register calculation, we assume the parameters of the freestanding quantum dot pillar structure (shown in Fig. 1) as follows: The height of the pillar is taken to be $\mathcal{L}=1000 \mathrm{~nm}$ while the radii of the core and sheath are taken to be $R_{c}=7 \mathrm{~nm}$ and $R=50 \mathrm{~nm}$. The drain and source contacts at the top and bottom of the pillar are grounded and a cylindrical gate with a height of $400 \mathrm{~nm}$ is placed around the center of the pillar. Near the source and drain contacts, layers of intrinsic semiconductor serve to inhibit gate-to-source and gate-to-drain currents. The central $600 \mathrm{~nm}$ of the pillar is uniformly n-doped with a doping concentration of $N_{D}=5 \times 10^{17} \mathrm{~cm}^{-3}$.

The cylindrical sheath surrounding the core region is composed of high band gap $A l_{0.45} G a_{0.55} A s$ and serves to confine electrons to the core region. The three qubits in the core are defined by the composition profile of $A l_{x} G a_{1-x} A s$ along the pillar axis. In our structure, the $\mathrm{Al}$ concentration, $x$, in the core region is uniform in the radial direction. The composition profile along the pillar axis in the core region is shown in Fig. 8. The ground and first excited electronic states are the qubit states $|0\rangle$ and $|1\rangle$ and the electron charge densities for these states are shown schematically in the figure. We find that in thermal equilibrium the electrons reside entirely in the ground state $|0\rangle$ for temperatures as high as $77 K$ since the energy gap between $|0\rangle$ and $|1\rangle$ is much greater than $k T$. This is indicated 
schematically by the solid circles in the diagram. The widths $L$ of the asymmetric quantum wells/dots defining qubits 1 through 3 are 19.0, 20.5 and $22.0 \mathrm{~nm}$ while the $B / L$ ratios are $0.670,0.683$ and 0.675 respectively. Our $1 \mathrm{D}$ analysis suggests that asymmetry parameters in this range will result in long spontaneous emission lifetimes and strong dipole-dipole coupling between neighboring qubits. The asymmetric quantum wells are composed of GaAs and $A l_{0.2} G a_{0.8} A s$ layers and the barriers between the asymmetric dots/wells are composed of $A l_{0.45} G a_{0.55} A s$.

With a properly chosen reverse gate bias, $V_{g}$, the doping region in the center of the pillar is depleted and the equilibrium Fermi level lines up so that there is exactly one electron in each dot. Single electron occupancy in the dots is necessary in order for there to be a well defined qubit Hilbert space. Due to shell filling effects, single electron occupancy in all three dots holds over a finite range of the gate voltage. By running our device model for several values of $V_{g}$, we find that single electron occupancy is obtained over the range $-1.56 \mathrm{~V} \leq V_{g} \leq-1.48 \mathrm{~V}$. Thus, the requirement for single electron occupancy in the quantum dots is maintained in the presence of gate voltage fluctuations on the order of $\Delta V_{g} \approx 0.08 \mathrm{~V}$. For $V_{g}=-1.5 \mathrm{~V}$, the self-consistent electron potential along the pillar axis, (i.e. $\rho=0$ ) is shown in Fig. 9 as a function of position along the pillar axis. The position along the pillar axis is measured from the drain contact at $z=0 \mathrm{~nm}$ to the source contact at $z=1000 \mathrm{~nm}$. Figure 9 is centered on the active region of the register containing the three quantum dots and the origin of the energy scale is chosen to be the equilibrium Fermi level. The total electron potential is approximately the sum of the self-consistent electrostatic Hartree potential and the conduction band offset potential, the self-consistent exchange-correlation potential being negligible.

The self-consistent electron levels are obtained by solving the Schrödinger equation in the self-consistent potential shown in Fig. 9. In our structure, the $|0\rangle$ ground states have $\left(n_{\rho}, n_{\phi}, n_{z}\right)=(0,0,0)$ symmetry and the $|1\rangle$ states (the first excited level) in all three qubits are $\left(n_{\rho}, n_{\phi}, n_{z}\right)=(0,0,1)$ states. The self-consistent qubit energy gap, $\Delta E_{0}$, between the $|0\rangle$ and $|1\rangle$ states, the radial energy gap, $\Delta E_{1}$, and the spontaneous emission lifetime of 
the $|1\rangle$ state, $\tau_{s}$, and dipole moment, $d$, for the three qubits are listed in Table $\llbracket$. From Table 【, we see that the radial energy gaps are larger than the qubit energy gaps. Another thing to note is that the qubit energy gaps are large compared to $k T$ at $T=77 \mathrm{~K}$. Thus, in thermal equilibrium the electrons reside entirely in the $|0\rangle$ level at $77 \mathrm{~K}$. This means that the initial state of the quantum register is characterized by a pure state density matrix $\hat{\rho}_{0}=|0,0,0\rangle\langle 0,0,0|$. Consequently, there is no need for initial state preparation in our quantum register. In Fig. 10, the self-consistent electron probability densities in the three quantum dots are plotted as a function of position along the pillar axis. Each dot traps one electron and the probability densities in the ground and first excited states are shown as solid and dot-dashed lines, respectively. The barriers are thick enough so that electron wavefunctions in adjacent dots do not overlap.

The energy levels for the three qubit quantum computer are shown in Table $\mathbb{I}$ with and without the inclusion of dipole-dipole coupling between the qubits. From Table III we see that a different energy corresponds to each three-electron state $\left|i_{1}, i_{2}, i_{3}\right\rangle$ of the register where $i_{n}=(0,1)$ labels the state of the $n$-th qubit. Transition energies between the states $|0\rangle$ and $|1\rangle$ for a given qubit are obtained by taking differences between the appropriate entries in Table ㄸ. F. For the first qubit, we take differences between all three-particle states $\left|0, i_{2}, i_{3}\right\rangle$ and $\left|1, i_{2}, i_{3}\right\rangle$. In general, the transition energy between $|0\rangle$ and $|1\rangle$ for an electron in the first qubit will depend on the states, $i_{2}$ and $i_{3}$, occupied by the second and third qubits, and there can be as many as four such conditional transitions. In the absence of dipole-dipole coupling between qubits, all four conditional transition energies between $|0\rangle$ and $|1\rangle$ for a given qubit are degenerate. When dipole-dipole interactions between the qubits are considered, the four-fold degenerate conditional transition energies split into multiplets depending on which states are occupied by the electrons in neighboring qubits.

The conditional transition energies between $|0\rangle$ and $|1\rangle$ states for our three qubit register are shown in Fig. 11 as a function of photon energy. In the absence of dipole-dipole coupling, the transition energies for the three qubits are $40.86 \mathrm{meV}, 47.14 \mathrm{meV}$, and $52.31 \mathrm{meV}$, respectively. When dipole-dipole interactions between qubits are taken into account, the 
conditional transition energies split into multiplets as shown in this figure. Each transition in the spectrum is labeled by the neighboring electron states which give rise to it. By performing optical $\pi$-pulses at selected conditional transition frequencies, quantum logic operations can be performed. For example, a $\pi$ pulse performed on the lowest energy transition in Fig. 11 performs a bit flip on the first qubit provided the second qubit is in state $|1\rangle$. This operation is just a Controlled-Not gate with qubit 2 as the control bit and qubit 1 as the target bit.

The need to selectively perform $\pi$-pulses at the conditional transition frequencies allows us to make some preliminary estimates on the parameters of the laser system needed to drive a quantum computation. If we want to selectively drive a given transition without exciting neighboring transitions, then the bandwidth of the $\pi$-pulse needs to be less than the splitting between the two most closely spaced lines in the spectrum. From Fig. 11, the two most closely spaced lines are spaced $\Delta \hbar \omega \approx 0.0776 \mathrm{meV}$ apart. If we require that the $\pi$-pulse bandwidth is $\Delta E_{\pi} \approx 0.01 \mathrm{meV}$, then the pulse length can be estimated from Heisenberg's uncertainty principle, $\Delta E_{\pi} \Delta T_{\pi} \approx \hbar / 2$, to be $T_{\pi} \approx 33 \mathrm{ps}$. If we assume a square $\pi$-pulse, the magnitude of the optical electric field is given by 37

$$
E_{0}=\frac{\pi \hbar}{q d T_{\pi}}
$$

where $d$ is the optical dipole from Table 1 and the average Poynting vector during the pulse is 38

$$
S_{a v}=\frac{1}{2} c \epsilon_{0} E_{0}^{2}
$$

For $d \approx 10 \AA$ and $T_{\pi} \approx 33 \mathrm{ps}$, we obtain $E_{0} \approx 0.627 \mathrm{kV} / \mathrm{cm}$ and $S_{a v} \approx 522 \mathrm{~W} / \mathrm{cm}^{2}$.

\section{SUMMARY}

In this paper, we have studied a solid state implementation of quantum computing based

on coupled quantum dots. Our quantum register consists of a free standing n-type pillar 
with grounding leads at the top and bottom of the structure. Asymmetric quantum wells confine electrons along the pillar axis and a high bandgap AlGaAs sheath wrapped around the center of the pillar allows for confinement in the radial direction. The ground and first excited electronic states of the quantum dots act as qubit states $|0\rangle$ and $|1\rangle$, respectively. We have developed a 3D device model for a general SET structure containing several quantum dots. We self-consistently solve coupled Schrödinger and Poisson equations for the device and develop a design for a three qubit quantum register with asymmetric quantum dots tailored for long dephasing time and large dipole-dipole coupling between the dots. Our

results indicate that a single gate electrode can be used to localize a single electron in each of the quantum dots. Adjacent dots are strongly coupled by electric dipole-dipole interactions arising from the dot asymmetry thus enabling rapid computation rates.

\section{ACKNOWLEDGMENTS}

This study was supported, in part, by the Defense Advanced Research Project Agency and the Office of Naval Research. 


\section{REFERENCES}

${ }^{1}$ L. K. Grover, Phys. Rev. Lett. 79, 325 (1997).

${ }^{2}$ P. W. Shor, "Algorithms for quantum computation: Discrete logarithms and factoring," in Proceedings of the 35th Annual IEEE Symposium on Foundations of Computer Science (IEEE Computer Society Press, Los Alamitos, CA, 1994), pp. 124-134.

${ }^{3}$ J. I. Cirac and P. Zoller, Phys. Rev. Lett. 74, 4091 (1995); C. Monroe, D. M. Meekhof, B. E. King, W. M. Itano, and D. J. Wineland, Phys. Rev. Lett. 75, 4714 (1995).

${ }^{4}$ T. Pellizzari, S. A. Gardiner, J. I. Cirac, and P. Zoller, Phys. Rev. Lett. 75, 3788 (1995); Q. A. Turchette, C. J. Hood, W. Lange, H. Mabuchi, and H. J. Kimble, Phys. Rev. Lett. 75, $4710(1995)$.

${ }^{5}$ I. L. Chuang, N. Gershenfeld, and M. Kubinec, Phys. Rev. Lett. 80, 3408 (1998); D. G. Cory, M. D. Price, W. Maas, E. Knill, R. Laflamme, W. H. Zurek, T. F. Havel, and S. S. Somaroo, Phys. Rev. Lett. 81, 2152 (1998).

${ }^{6}$ A. Shnirman, G. Schön, and Z. Hermon, Phys. Rev. Lett. 79, 2371 (1997).

${ }^{7}$ N. J. Cerf, C. Adami, and P. G. Kwiat, Phys. Rev. A 57, R1477 (1998).

${ }^{8}$ A. Barenco, D. Deutsch, A. Ekert, and R. Jozsa, Phys. Rev. Lett. 74, 4083 (1995).

${ }^{9}$ A. A. Baladin and K. L. Wang, private communication.

${ }^{10}$ B. E. Kane, Nature 393, 133 (1998).

11 T. Tanamoto, e-print quant-ph/9902031.

12 D. Loss and D. P. DiVincenzo, Phys. Rev. A 57, 120 (1998); G. Burkard, D. Loss, and D. P. DiVincenzo, Phys. Rev. B 59, 2070 (1999).

${ }^{13}$ S. Tarucha, D. G. Austing, T. Honda, R. J. van der Hage, and L. P. Kouwenhoven, Phys. Rev. Lett. 77, 3613 (1996). 
${ }^{14}$ D. Collins, K. W. Kim, W. C. Holton, H. Sierzputowska-Gracz, and E. O. Stejskal, e-print quant-ph/9910006.

${ }^{15}$ C. Leichtle, W. P. Schleich, I. Sh. Averbukh, and M. Shapiro, Phys. Rev. Lett. 80, 1418 (1998); T. C. Weinacht, J. Ahn, and P. H. Bucksbaum, Phys. Rev. Lett. 80, 5508 (1998).

${ }^{16}$ M. S. Sherwin, A. Imamoglu, and T. Montroy, Phys. Rev. A 60, 3508 (1999).

${ }^{17}$ A. Mekis, J. C. Chen, I. Kurland, S. Fan, P. R. Villeneuve, and J. D. Joannopoulos, Phys. Rev. Lett. 77, 3787 (1996).

${ }^{18}$ D. G. Cory, M. D. Price, and T. F. Havel, Physica D 120, 82, (1998); D. G. Cory, A. E. Dunlop, T. F. Havel, S. S. Somaroo, and W. Zhang, e-print quant-ph/9809045.

${ }^{19}$ D. V. Averin, A. N. Korotkov, and K. K. Likharev, Phys. Rev. B 44, 6199 (1991).

${ }^{20}$ M. Stopa, Phys. Rev. B 48, 18340 (1993).

${ }^{21}$ M. Macucci, K. Hess, and G. J. Iafrate, Phys. Rev. B 48, 17354 (1993).

${ }^{22}$ D. Jovanovic and J. P. Leburton, Phys. Rev. B 49, 7474 (1994).

${ }^{23}$ M. Stopa, e-print cond-mat/9609015.

${ }^{24}$ G. Todorovic, V. Milanovic, Z. Ikonic, and D. Indjin, Phys. Rev. B 55, 15681 (1997).

${ }^{25}$ M. Macucci, K. Hess, and G. J. Iafrate, Phys. Rev. B 55, R4879 (1997).

${ }^{26}$ S. Nagaraja, P. Matagne, V. Y. Thean, J. P. Leburton, Y. H. Kim, and R. M. Martin, Phys. Rev. B 56, 15752 (1997).

${ }^{27}$ L. R. C. Fonseca, J. L. Jimenez, J. P. Leburton, and R. M. Martin, Phys. Rev. B 57, 4017 (1998).

${ }^{28}$ J. I. Pankove, Optical Processes in Semiconductors (Dover, New York, 1975).

${ }^{29}$ H. A. Bethe and R. Jackiw, Intermediate Quantum Mechanics (W. A. Benjamin, Reading, 
MA, 1968).

${ }^{30}$ H. J. Lee, L. Y. Jurovel, J. C. Wolley, and A. J. Springthorpe, Phys. Rev. B 21, 659 (1980).

31 J. P. Perdew and Alex Zunger, Phys. Rev. B 23, 5048 (1981).

${ }^{32}$ L. Jacak, P. Hawrylak, and A. Wòjs, Quantum Dots (Springer-Verlag, New York, 1998).

33 T. Inoshita and H. Sakaki, Phys. Rev. B 46, 7260 (1992).

${ }^{34}$ U. Bockelman and G. Bastard, Phys. Rev. B 42, 8947 (1990).

${ }^{35}$ H. Benisty, Phys. Rev. B 51, 13281 (1995).

${ }^{36}$ A. Ekert and R. Jozsa, Rev. Mod. Phys. 68, 733 (1996).

${ }^{37}$ G. Mahler and V. A. Weberruss, Quantum Networks: Dynamics of Open Nanostructures (Springer-Verlag, New York, 1998).

${ }^{38}$ P. Lorraine and D. Corson, Electromagnetic Fields and Waves (W. H. Freeman, San Francisco, 1970). 


\section{TABLES}

TABLE I. Self-consistent qubit energy gap, $\Delta E_{0}$, radial energy gap, $\Delta E_{1}$, spontaneous emission lifetime, $\tau_{s}$, and optical dipole moment, $d$, for a three qubit quantum register.

\begin{tabular}{ccccc}
\hline \hline Qubit No. & $\Delta E_{0}(\mathrm{meV})$ & $\Delta E_{1}(\mathrm{meV})$ & $\tau_{s}(n s)$ & $d(\AA)$ \\
\hline 1 & 40.86 & 63.7 & 28000 & 11.7 \\
2 & 47.14 & 63.2 & 19000 & 11.5 \\
3 & 52.31 & 61.9 & 14000 & 11.4 \\
\hline \hline
\end{tabular}

TABLE II. Register energies for a three qubit quantum register, with and without dipole-dipole coupling interaction between qubits.

\begin{tabular}{|c|c|c|}
\hline Register state & $\begin{array}{c}\text { Energy }(\mathrm{meV}) \\
\text { uncoupled }\end{array}$ & $\begin{array}{c}\text { Energy }(\mathrm{meV}) \\
\text { dipole-dipole coupled }\end{array}$ \\
\hline$\left|\begin{array}{lll}0 & 0 & 0\end{array}\right\rangle$ & -70.161 & -70.357 \\
\hline$\left|\begin{array}{lll}0 & 0 & 1\end{array}\right\rangle$ & -17.852 & -17.833 \\
\hline$\left|\begin{array}{lll}0 & 1 & 0\end{array}\right\rangle$ & -23.017 & -22.822 \\
\hline$\left|\begin{array}{lll}0 & 1 & 1\end{array}\right\rangle$ & 29.292 & 29.273 \\
\hline$\left|\begin{array}{lll}1 & 0 & 0\end{array}\right\rangle$ & -29.292 & -29.311 \\
\hline$\left|\begin{array}{lll}1 & 0 & 1\end{array}\right\rangle$ & 23.017 & 23.213 \\
\hline$\left|\begin{array}{lll}1 & 1 & 0\end{array}\right\rangle$ & 17.852 & 17.871 \\
\hline$\left|\begin{array}{lll}1 & 1 & 1\end{array}\right\rangle$ & 70.161 & 69.966 \\
\hline
\end{tabular}




\section{FIGURES}

FIG. 1. Schematic illustration of the proposed quantum dot pillar structure. As an example, the structure is shown for a three qubit quantum register (i.e., with three quantum dots in the middle).

FIG. 2. Probability density along the confinement direction, $z$, for the qubit wavefunctions $|0\rangle$ (solid line) and $|1\rangle$ (dot-dashed line). The inset shows a schematic illustration of the conduction bandedge profile in the $z$ direction.

FIG. 3. Transition energy, $\Delta E$, between $|0\rangle$ and $|1\rangle$ in an $L=20 \mathrm{~nm} \mathrm{GaAs} / A l_{x} G a_{1-x} A s$ asymmetric quantum dot as a function of $B / L$ for several values of $x$.

FIG. 4. The transition energy between the $|0\rangle$ and $|1\rangle$ qubit states as a function of the asymmetric quantum well width, $L$, for several values of the asymmetry parameter $B / L$ and fixed barrier Al concentration $x=0.2$. The continuous curves are based on a simple $1 \mathrm{D}$ analysis and the squares are the qubit energy gaps for quantum dots as determined by the self-consistent calculation described in the text.

FIG. 5. The energy gap between the $|0\rangle$ ground state and the lowest lying radial eigenstate

(with $\left(n_{\rho}, n_{\phi}, n_{z}\right)=(0,1,0)$ symmetry) as a function of the n-doping concentration, $N_{D}^{+}$. The radial energy splitting for several pillar radii. Greater confinement of the radial wavefunction can be obtained by decreasing the pillar radius (size confinement) or increasing the doping concentration (electrostatic confinement). In the limit of large pillar radii, the radial energy splitting is determined by confinement in a parabolic electrostatic potential.

FIG. 6. Dipole-dipole interaction between two asymmetric $G a A s / A l_{x} G a_{1-x} A s$ quantum dots of widths $L 1=19 \mathrm{~nm}$ and $L 2=21 \mathrm{~nm}$ separated by an $A l_{y} G a_{1-y} A s$ barrier of width $W b=10 \mathrm{~nm}$. The coupling energy is plotted as a function of $B / L$ for several values of $x . B / L$ and $x$ are the same for both dots. 
FIG. 7. Spontaneous emission lifetime for qubit state $|1\rangle$ in a $G a A s / A l_{x} G a_{1-x} A s$ quantum dot with $L=20 \mathrm{~nm}$ as a function of $B / L$ for several values of $x$.

FIG. 8. Composition profile along the pillar axis for a three qubit quantum register.

FIG. 9. Self consistent electron potentials as a function of position along the pillar axis in the active region of a three qubit quantum register. The total potential (solid line) is the sum of the Hartree potential (dot-dashed line) and the band offset potential. The fermi level (dotted line) is aligned so that exactly one electron resides in each quantum dot.

FIG. 10. Electron density as a function of position along the pillar axis in the active region of a three qubit quantum register. The bias voltage is adjusted so that exactly one electron resides in the ground state of each quantum dot. In each dot, the probability density of the ground states (solid line) are shown along with the probability density of the first excited states (dot-dashed line). Depending on which state the electrons occupy, the electric dipole moment can point either left or right.

FIG. 11. Conditional transition energies between the qubit states $|0\rangle$ and $|1\rangle$ as a function of photon energy for a three qubit quantum register. In the absence of dipole-dipole coupling, the transition energies are $40.86 \mathrm{meV}, 47.14 \mathrm{meV}$, and $52.31 \mathrm{meV}$ respectively. When dipole-dipole interactions between qubits are taken into account, the transition energies depend on the state of the adjacent qubits. 
Sanders et al, Fig. 1

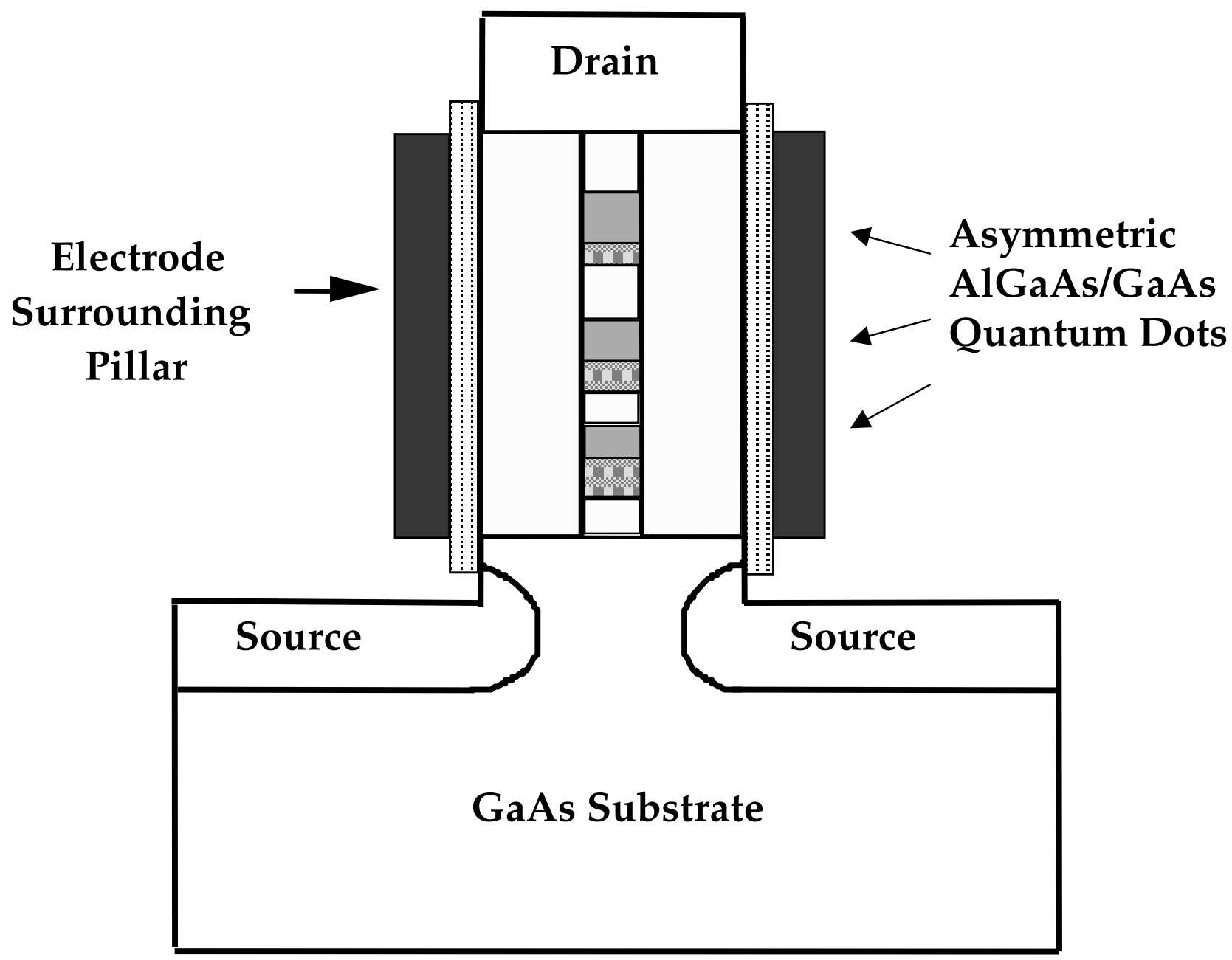


Sanders et al, Fig 2

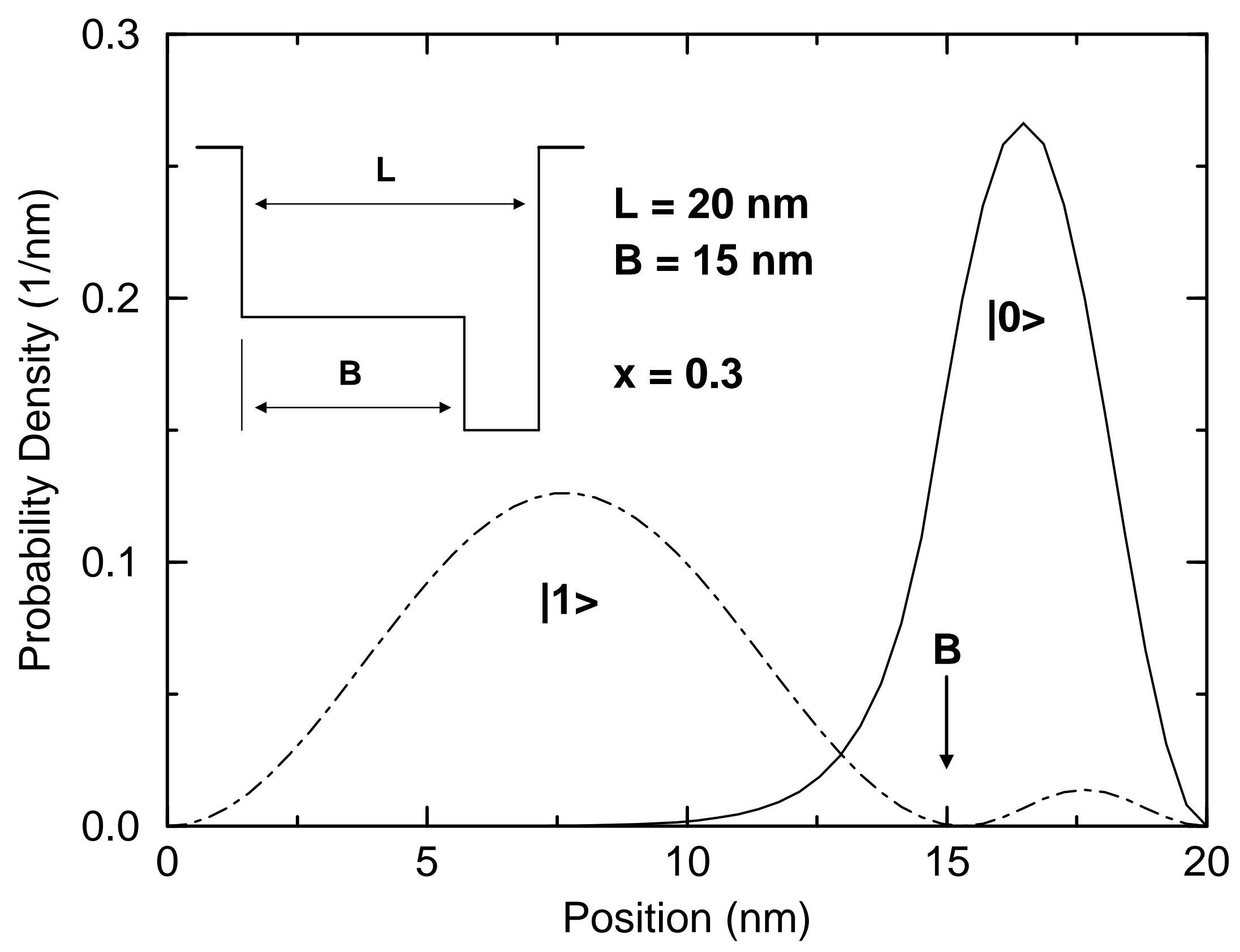


Sanders et al, Fig. 3

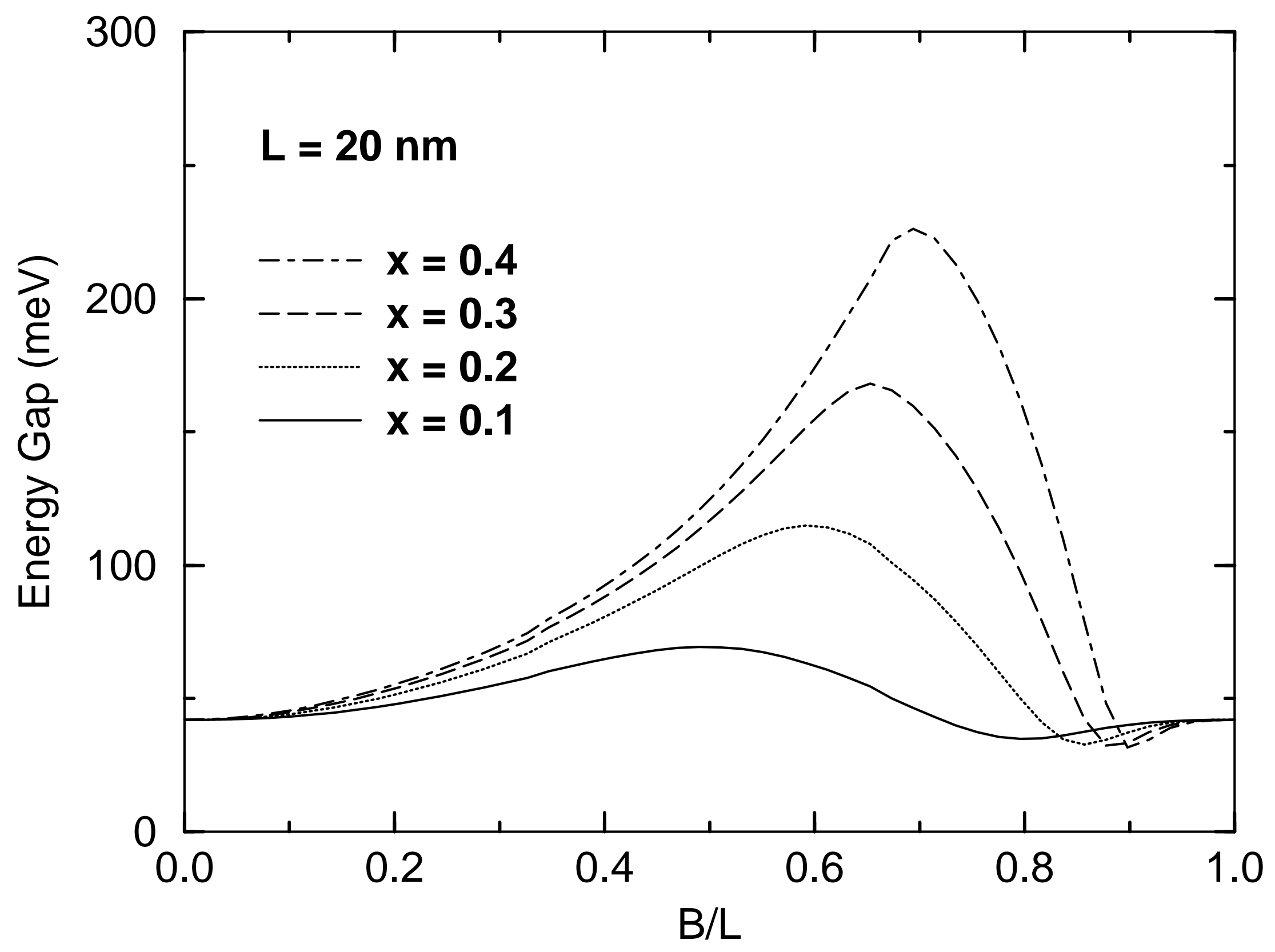


Sanders et al, Fig 4

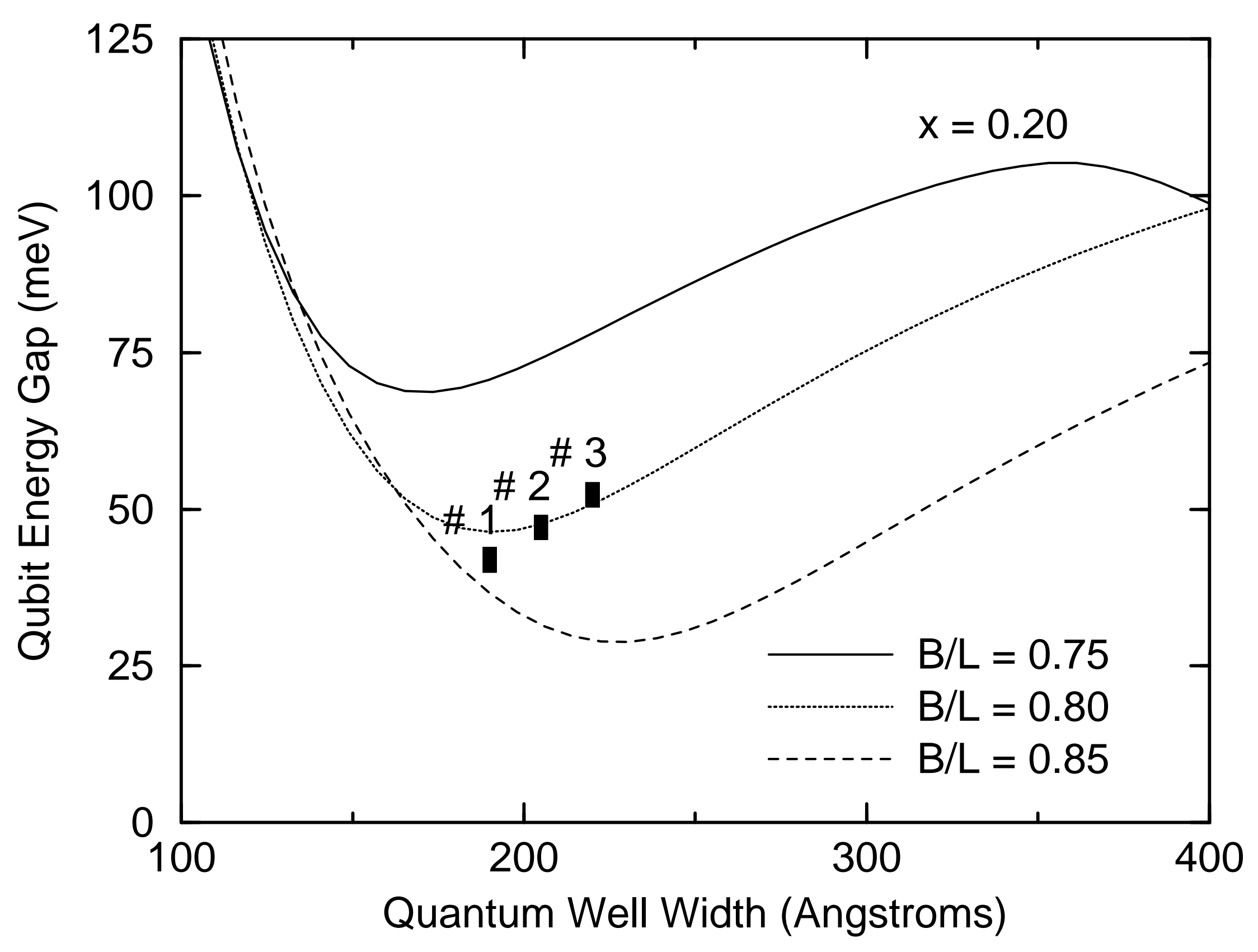


Sanders et al, Fig 5

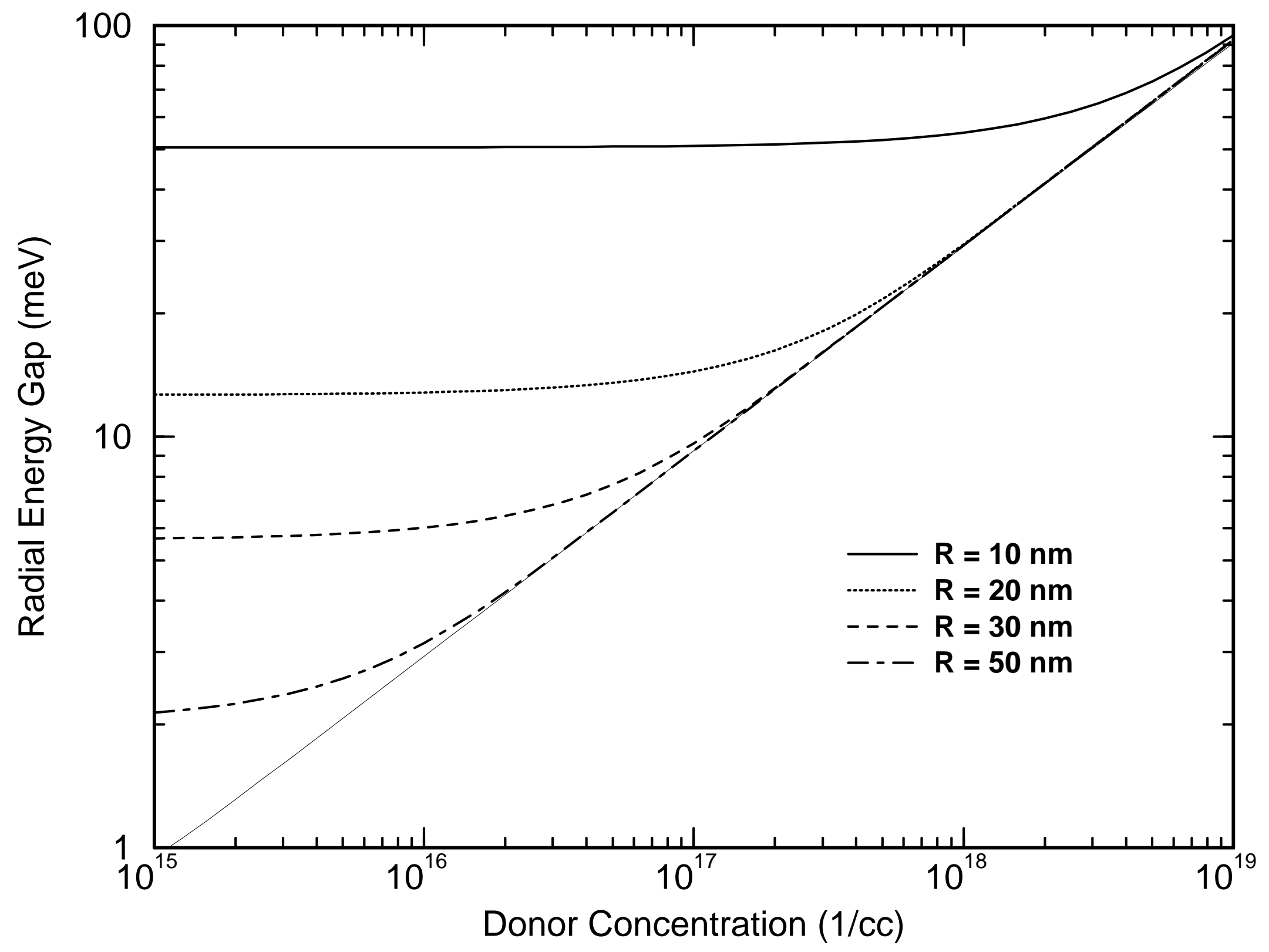


Sanders et al, Fig. 6

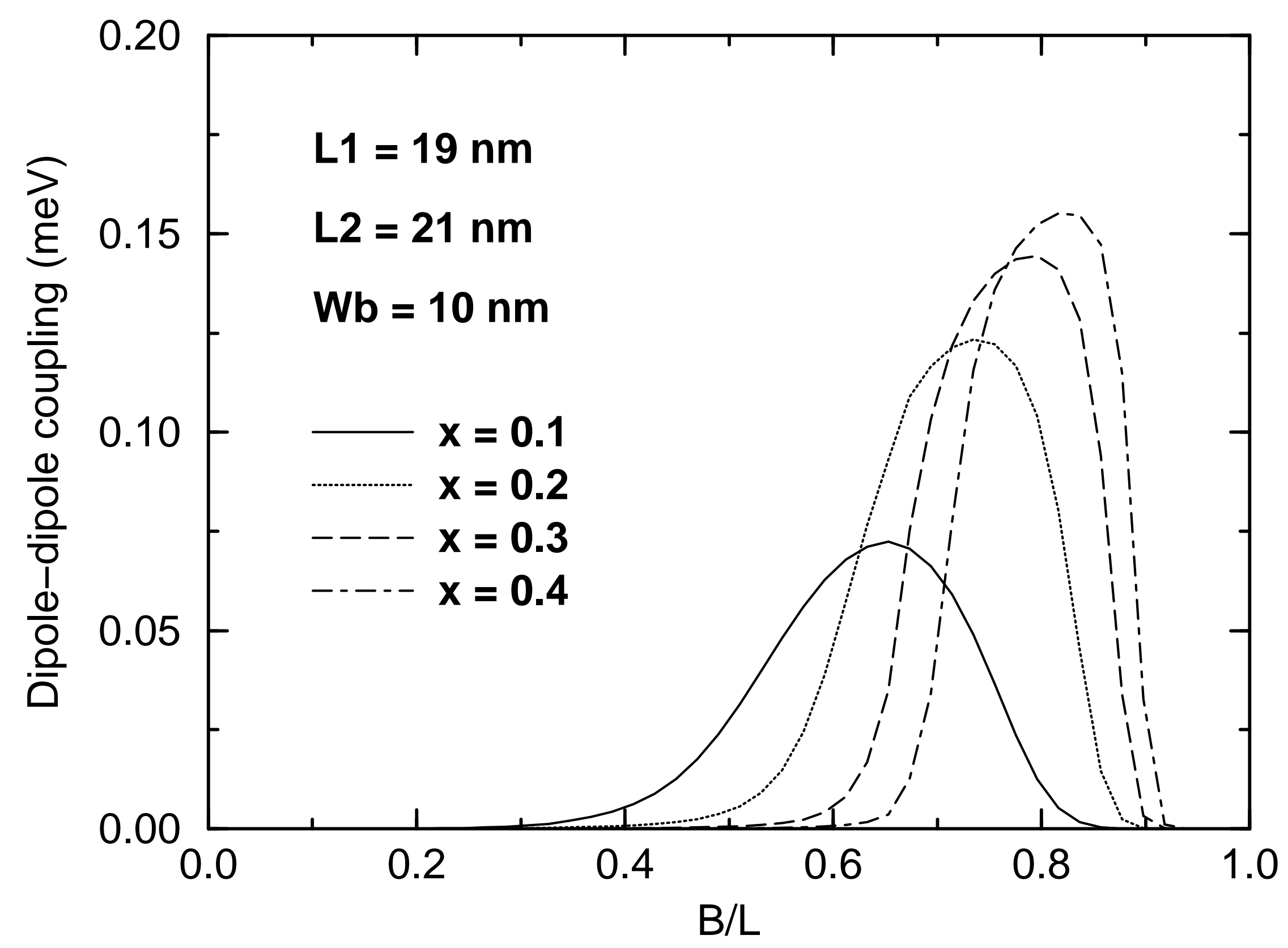


Sanders et al, Fig. 7

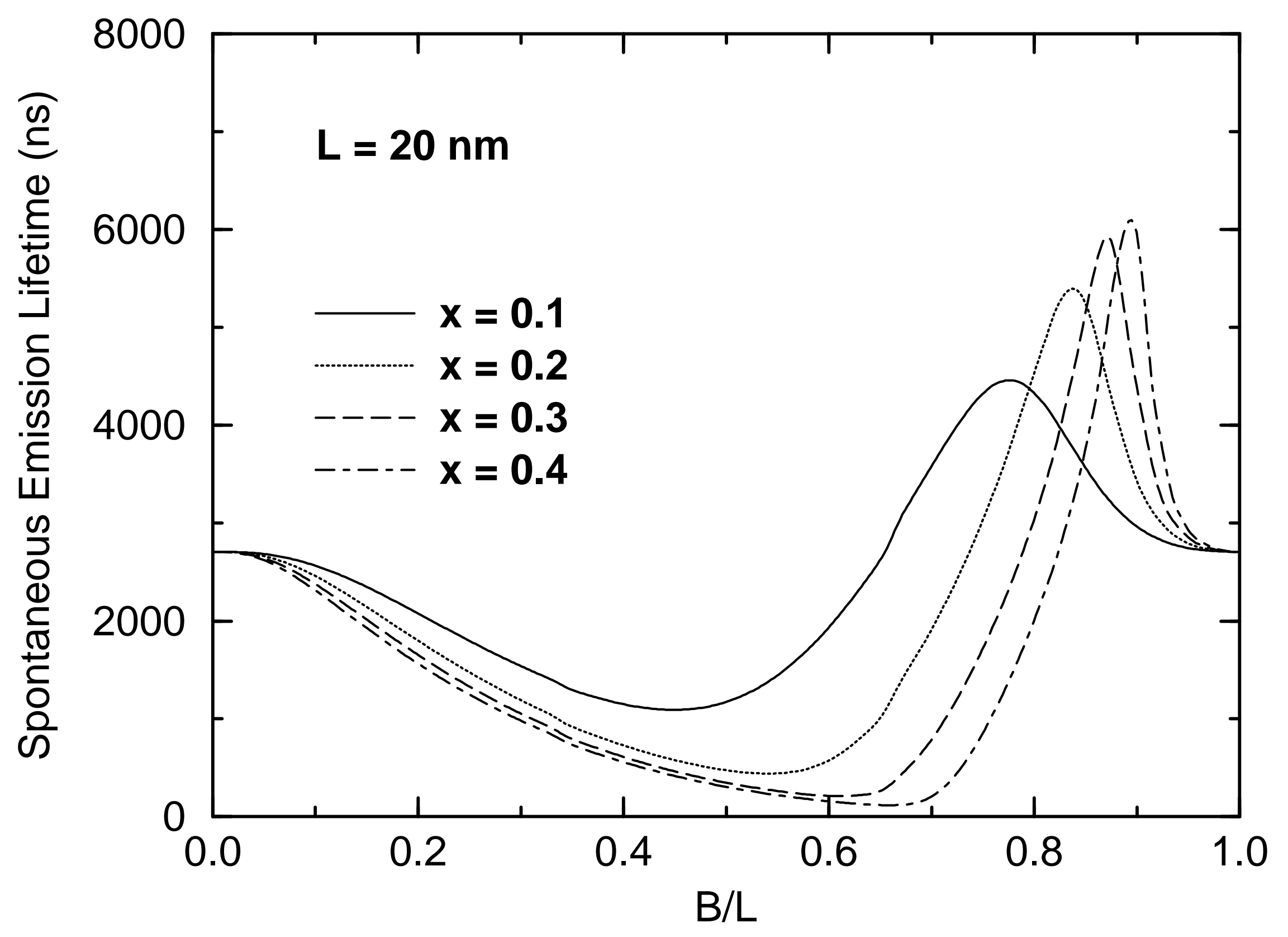




\section{Composition Profile along Pillar Axis}

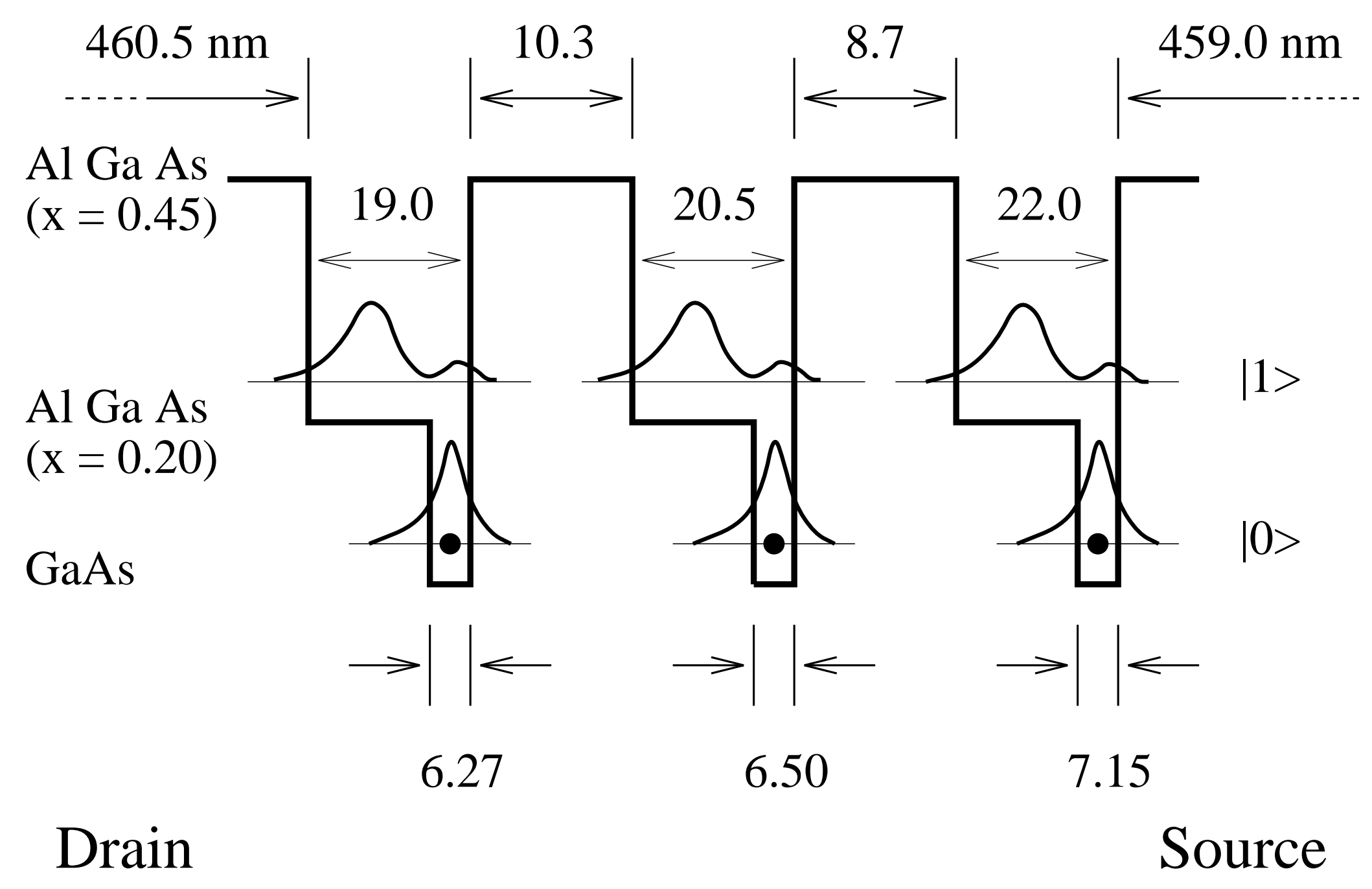


Sanders et al, Fig 9

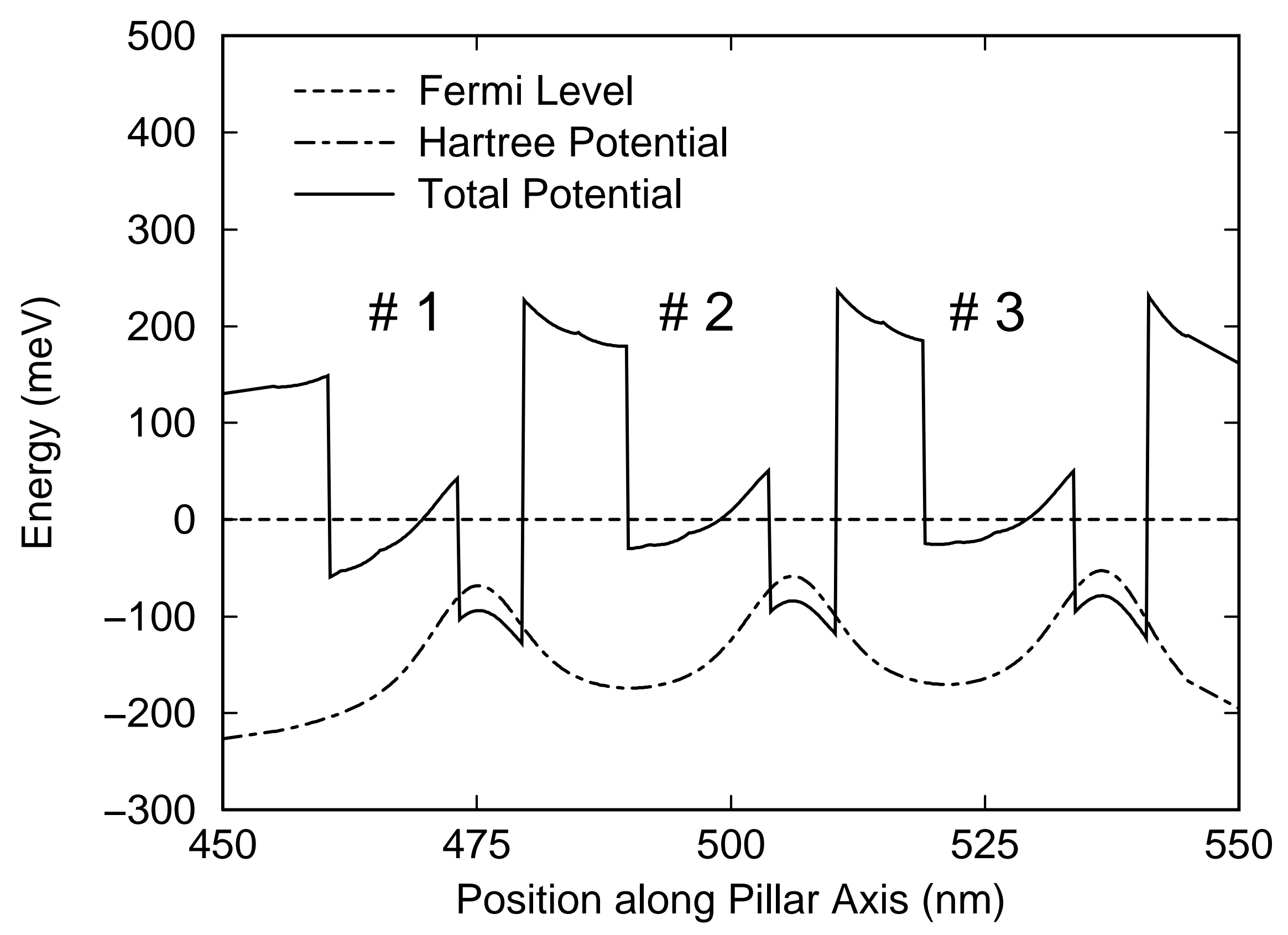


Sanders et al, Fig 10

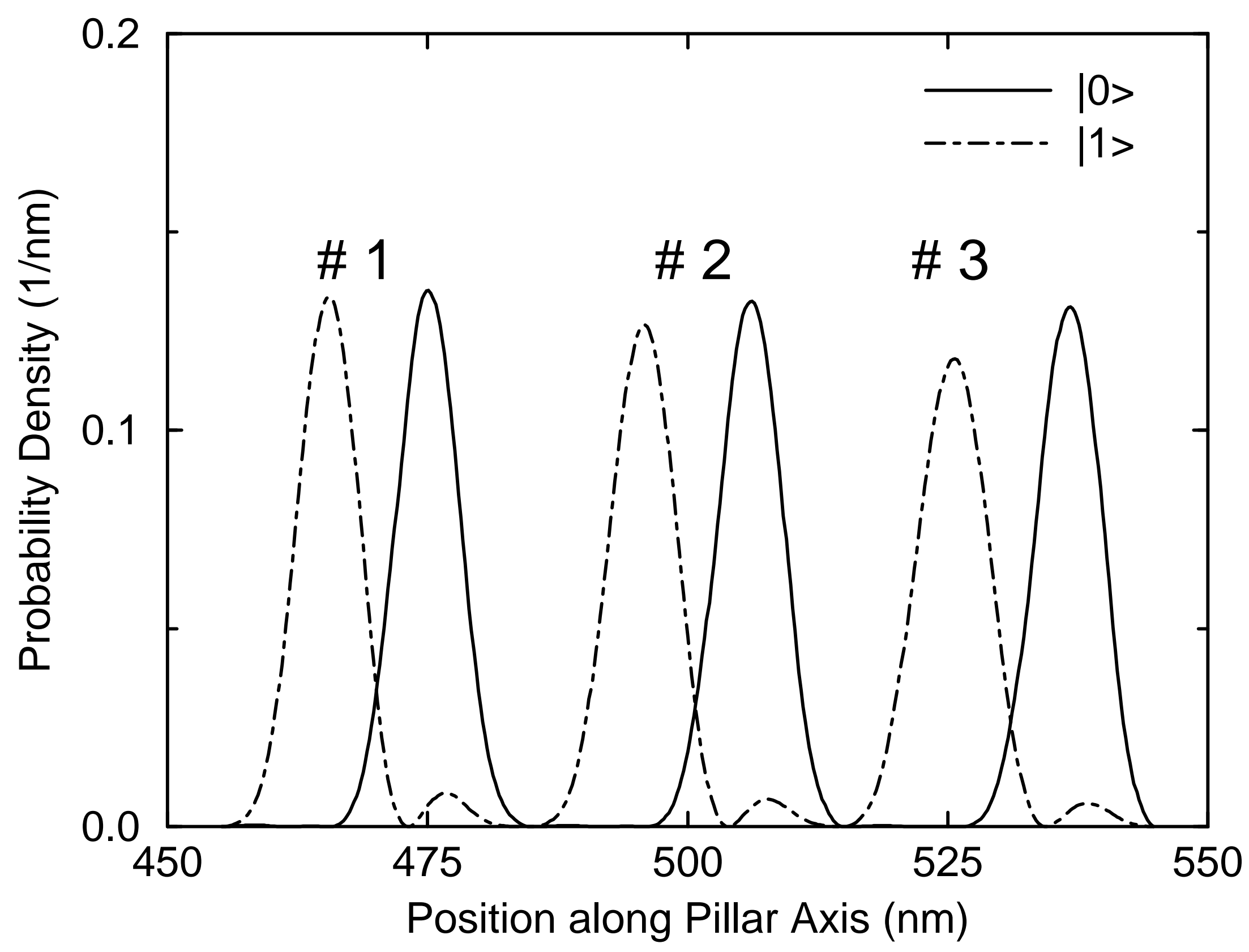




\section{Conditional Transition Energies}

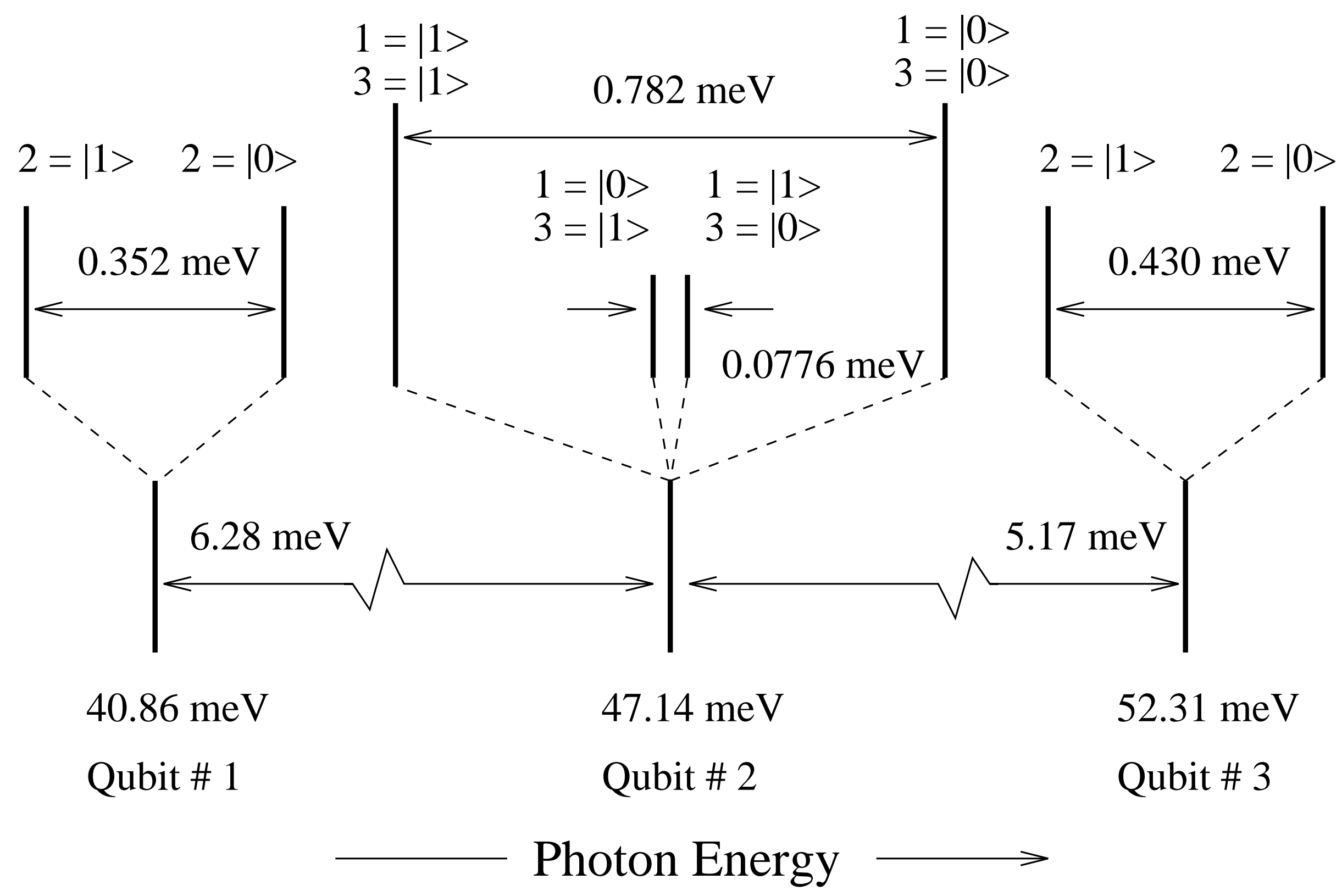

